\title{
Automatic Control Technology of Data Exchange Platform Based on Abnormal Traffic Monitoring in Cross Security Domain
}

\author{
Zhang Qing ${ }^{1, a}$,Zheng CuiFang ${ }^{2, b}$, and Cheng Zheng ${ }^{3, c}$ \\ ${ }^{1}$ Institute Computer Application, Chinese Academy of Engineering Physics,Mianyang Sichuan P.R. \\ China. \\ ${ }^{2}$ Institute Computer Application, Chinese Academy of Engineering Physics,Mianyang Sichuan P.R. \\ China. \\ azhangq@caep.ac.cn b zcfkb@sina.com,, chenengz@caep.ac.cn
}

Keywords: Data exchange, Star structure, Traffic early warning, Automatic blocking.

Abstract. Using message queue technology in the data exchange of star topology can solve the point-to-point reliable data transmission, it makes the data exchange platform has good expansibility and maintainability, but this existe a problem,that is any local failure may lead to "traffic jam" of central node. In order to solve this problem, in this paper, the data exchange platform mechanism of traffic monitoring and early warning and automatic control are design and achieve, by switching nodes, status monitoring of application system, traffic early warning, automatic blocking and other functions to avoid local failure lead to global paralysis problem, automatic blocking function to avoid partial failure. It improves the reliability, availability and flexibility of data exchange in complex networks, and has practical value.

\section{Introduction}

From the 90's of last century, with the rapid development of network technology, Data exchange technology has experienced several stages. In order to realize the communication between distributed systems, The RPC as the representative of the remote call technology is appeared. Implement point to point communication for system to system, with the development of CORBA and DCOM Technology, service based on data exchange technology start using. Into twenty-first Century, application integration technology which is the present of service oriented architecture (SOA) theory is rapid development, data exchange technology using message oriented middleware as core technology has been widely used, greatly promote the integration of large distribution systems.

In the design of data exchange platform using star topology can achieve unified control very well, and the message queue is used for data transmission between nodes, which can achieve reliable transmission of data, but, in practical use, it is found that there is a problem of local blocking cause global blocking. In order to solve the problem, we design and implement a data exchange platform for traffic monitoring and automatic control mechanism, Automatic blocking function is realized through the queue depth warning mechanism, it improves the reliability, availability and flexibility of data exchange in complex networks.

\section{Research on data exchange platform}

\section{Data exchange topology analysis}

Usually there are two kinds of communication network topology about the data exchange, one is star structure and another is network structure, the main difference between the network and the star structure in the data exchange node position. As shown below: 


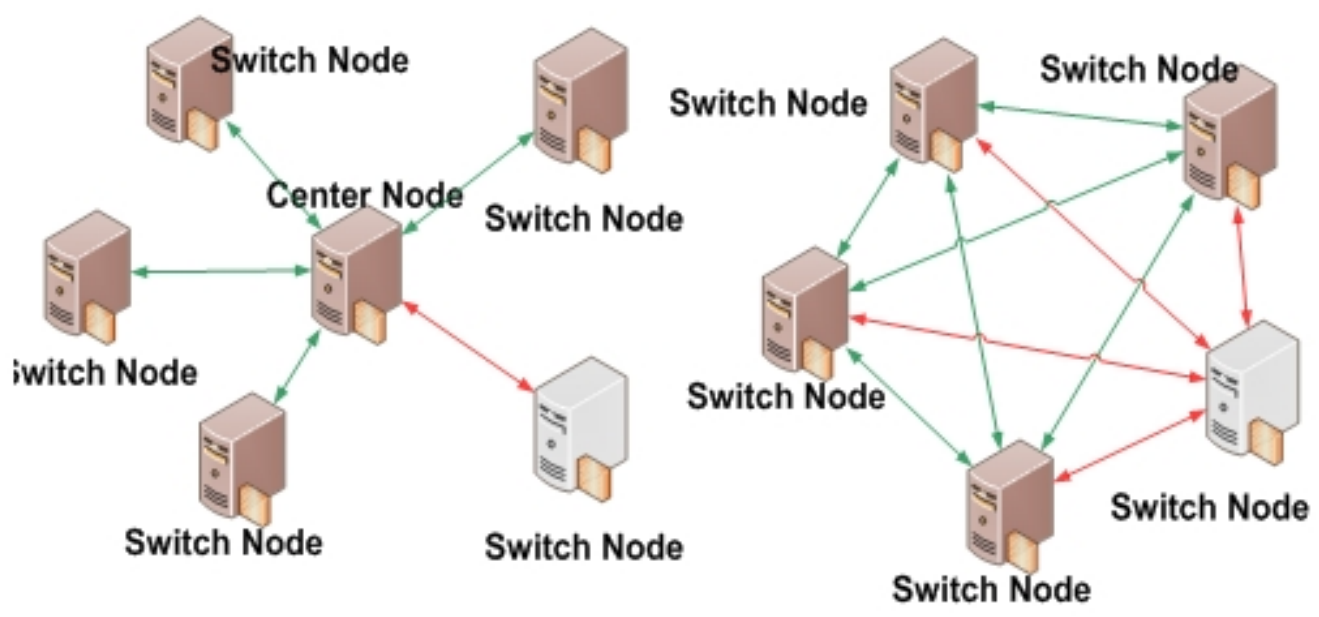

Fig.1 Comparison of star structure and network structure

In this paper, we use the star structure; Data exchange takes place on a central node. Through the data exchange center node, you can create a transparent and secure data channel between the data source and destination; Compared with the network structure has the following advantages:

1) The data exchange data of Star structure through the central node, this is conducive to centralized control and unified exchange standards;

2) the access and operation strategy of Star structure switching node is relatively simple, An access change of a node involves only the change between the central node and the center node, and each node in the network structure is tightly coupled,security policy configure complex, and safety management risk is higher, these increase the costs of system implementation and maintenance

3) Star data exchange structure, apart from being able to achieve unified management and control, it also has good expansibility and maintainability. Any change in the access node is only involved in the change of the node and the center node, the structure is more stable and efficient.

\section{Message Queuing transmission}

At present, the way of data exchange in the industry mostly adopts asynchronous communication mode, using the message queue technology to ensure reliable data transmission, a message queue is used for data transmission among each message queuing node(referred to as:MQN) in a star switching network.

Message queuing is a loosely coupled and reliable network communication service based on transaction model. Queue is a container which storage of routing messages temporary and saving messages in the message transmission. Message queue itself has the function of reliable transmission; each exchange node is a first in first out queue, transfer data to the next node when it is available. When the next MQN is not available, or when a fault occurs, the data can be cached until the target node is normal, Achieve fault recovery task, as shown in Fig.2

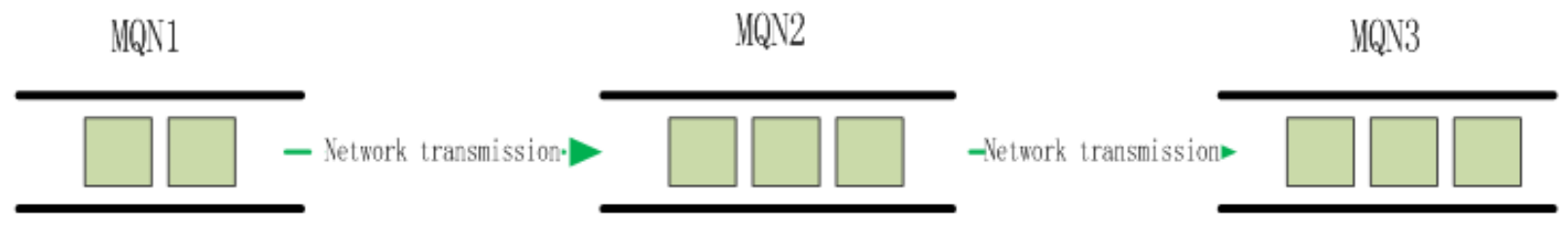

Fig. 2 Message Queuing transmission 
Traffic monitoring and early warning and automatic block design for cross domain data exchange platform

\section{Shortage of star structure data exchange}

Star switch structure is conducive to centralized management and control, but any local fault may cause chain reaction of blocking queue, once an exchange center node congestion occurs, the star exchange network will be unable to normal operation, as shown in Fig.3: when the MQN5 attached to a system failure to timely consumption news, resulting in the message of MQN5 stack blocking, And then cause the central queue block, When the center is fully filled with messages sent to the MQN5, and there is no space for receiving and distribution message of other node sending, this make the exchange platform paralysis. So just depend on the reliable transmission of the message queue itself is not enough.

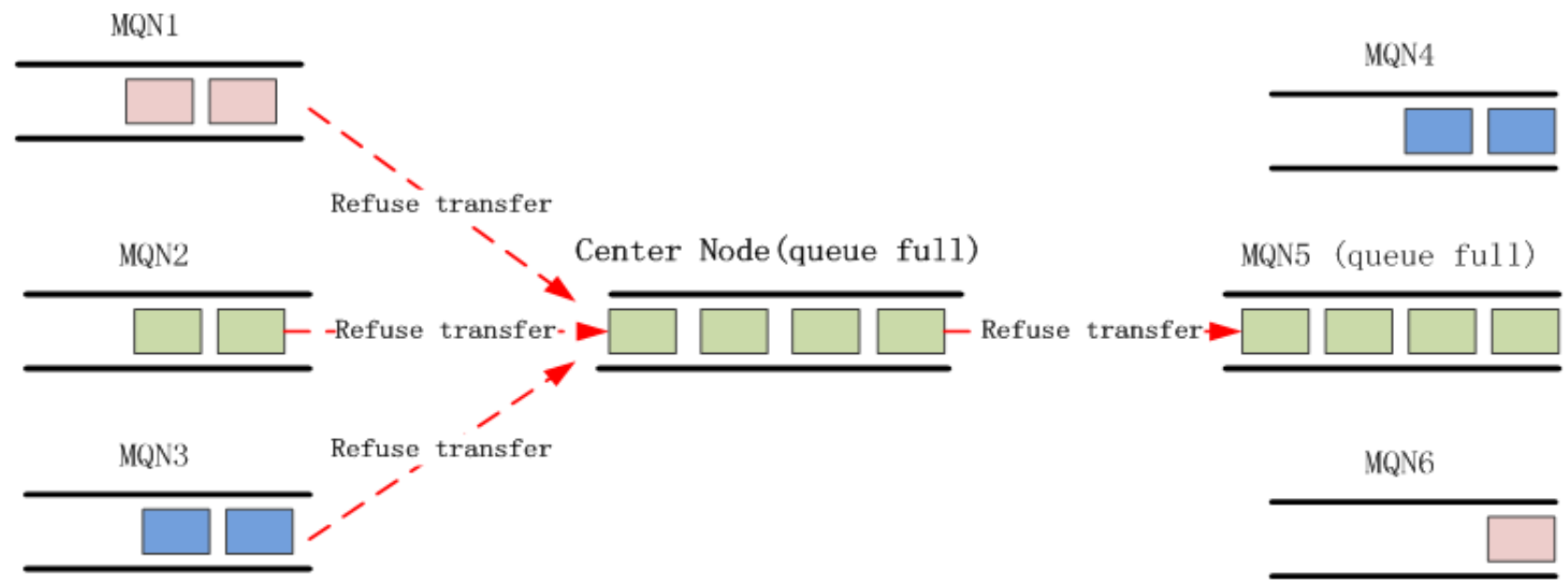

Fig.3 Schematic diagram of central node blocking

In order to solve the "traffic jam" problem of the central node, so as to ensure the normal operation of the whole data exchange network, the traffic monitoring and early warning and automatic blocking function are designed.

\section{Traffic monitoring and early warning and automatic blocking design}

Flow monitoring and early warning and automatic blocking is refers to When the number of messages to be processed in the queue reaches a certain number, the system will issue a warning, to remind other exchange nodes, temporarily reject all data exchange requests that are sent to the blocking node, thus avoiding the local failure lead to global paralysis. The specific working principle is shown in fig. 4.

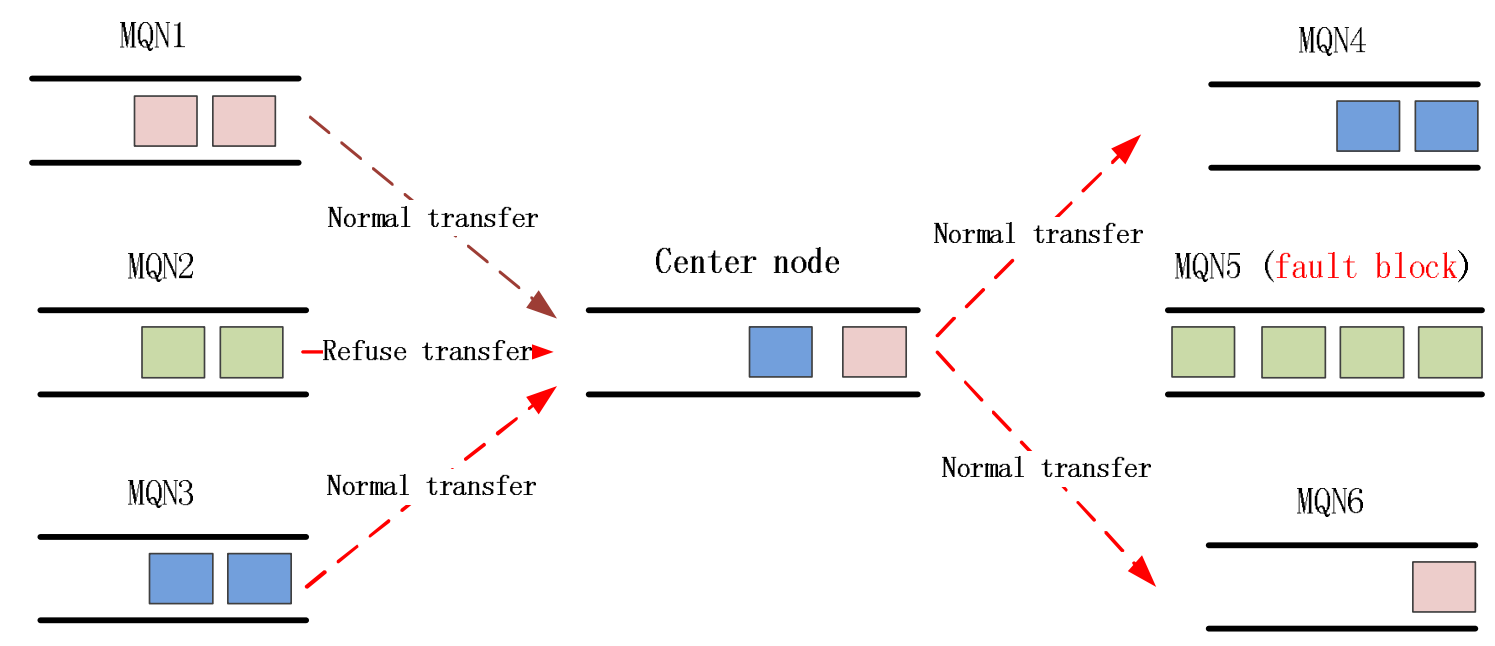

Fig.4 Traffic monitoring and early warning and automatic blocking 
When a local system failure or shutdown, the data sent to the system will be stacked in the queue of the exchange node, when queue depth reaches a predetermined value, the node will cause a node blocking message, and through the exchange platform to transfer to other switching nodes, each switching node will temporarily deny all data exchange requests that are sent to the blocking node, Guaranteed that the data of the target node will not be further blocked, and to ensure that the data exchange in the central queue can timely consumption. Therefore, other no fault nodes can still exchange data through the exchange center. When the system fault is restored and the data in the target node queue will be received by the application system, with the data in the queue is further reduced, queue depth is reduced to the alarm threshold; the current node will send a message to each node through the exchange platform, recovery data exchange and transmission of faulty nodes.

In this process, there are two key parameters, one is Qmax(Blocking threshold), another is Qmin(Obstruction threshold). The blocking event of this node is triggered when the queue message reaches the Qmax value, and the blocking state of the node is ended when the message is returned to the Qmin value. Among them Qmax>Qmin, Qmax derived from statistical data, Qmin is usually less than Qmin.In order to ensure that the target queue does not overflow, Qmax estimates are generally to meet: QL (queue capacity) -Qmax > Qin (the number of messages that have entered the platform but not arrive at this node); in this, Qin can be estimated based on the traffic statistics of data exchange platform.Under usual circumstances QL>Qmax >>Qin, but in fact, on the safe side, the value of Qmax is lower than the estimated value, in this project, QL=3000, $\max =2400, \mathrm{Qmin}=1800$. Qmax value should not be too low, if low, the asynchronous transfer capability under local fault will be reduced, this make premature blocking, and queue does not play a unique cache capacity.

\section{Conclusions}

Asynchronous communication technology based on message queue; solve the reliable point-to-point data transmission. in this paper, the early warning mechanism, it greatly improves operation efficiency of the whole data exchange platform on the basis of reliable asynchronous communication, the reliability of the distributed system in complex networks is reduced, flexibility and reliability improved. This has important reference value to improve the construction of large data exchange platform based on asynchronous communication.

In the future, we can study and design the node blocking and early warning mechanism,through automatic detection the nodes of the data exchange platform, and the application system connectivity, using dynamic and artificial combination method to adjust platform "traffic control status", So that the use efficiency of data exchange platform to further improve.

\section{Acknowledgements}

This work was supported by the Discipline Development foundation: No 13-FZjj-0143.

\section{References}

[1] Dj.M. Maric, P.F. Meier and S.K. Estreicher: Mater. Sci. Forum Vol. 83-87 (1992), p. 119

[1] OGSA-DAI WSRF2.2 http://www.ogsadai.org.uk/documentation/ogsadai-wsrf-2.2/doc/.

[2] LIU Jian-Xin, YAN Bao-Ping .Research on the architecture and key technology of OGSA-DAI [J].Computer Applications,2005,24 (11) : 81-87.

[3] OGSA-DAI Architecture http://www.ogsadai.org.uk/documentation/ogsadai-wsrf-2.2/doc/ background/architecture.html.

[4] Neil P Chue Hong,OGSA-DAI Overview. 
[5] [27] Interacting with Data Service Resources http://www.ogsadai.org.uk/documentation /ogsadai-wsrf-2.2/doc/interaction/index.html.

[6] LUO Qing-di,JIANG Xiu-feng.Research on Isomerous Data Integration Based on OGSA-DAI in Grid Environment[J].Computer Technology and Development,2009,19(3):144-147.

[7] GT4 Admin Guide,http://www.globus.org/toolkit/docs/4.0/admin/docbook/

[8] YANG Xue-Seng. Integrate Heterogeneous Database System Using OGSA-DAI Middleware [J]. Information Technology, 2011, 35(1):121-123. 